\title{
Co-infection, reinfection and superinfection with Anaplasma phagocytophilum strains in a cattle herd based on ankA gene and multilocus sequence typing
}

\author{
Denis B. Langenwalder ${ }^{1}$, Cornelia Silaghi ${ }^{2}$, Marion Nieder ${ }^{3}$, Martin Pfeffer $^{3}$ and Friederike D. von Loewenich ${ }^{{ }^{*}}$
}

\begin{abstract}
Background: Anaplasma phagocytophilum is a Gram-negative obligate intracellular bacterium that replicates in neutrophil granulocytes. It is transmitted by ticks of the Ixodes ricinus complex and causes febrile illness in humans and animals. We used multilocus sequence typing (MLST) and ankA gene-based typing to study the molecular epidemiology of the $A$. phagocytophilum strains circulating in a German cattle herd over one pasture season. The aim was to investigate whether co-infection with two distinct variants, reinfection with the same and/or superinfection by a different strain occurred during one pasture season. Eight genetic loci were sequenced in 47 PCR-positive samples from 15 animals.

Results: Five different sequence types (ST) and four ankA alleles were detected in the cattle herd. Three different ST caused clinically overt tick-borne fever in primary infected animals. The concordance between ST and ankA allele was 100\%. Therefore, the housekeeping genes used for MLST and the highly variable ankA gene were concatenated to increase resolution. Co-infection could be proven because samples of chronologically close collection dates were included. Co-infecting A. phagocytophilum strains differed by 14 to 18 single nucleotide polymorphisms (SNPs). Most superinfecting variants varied by 14 SNPs from the previous strain and appeared in median after a free interval of 31 days. Thus, it is unlikely that superinfecting strains arose by in-animal evolution. Immunity against re- or superinfection was assumed because the cattle developed clinical signs only during primary infection.
\end{abstract}

Conclusions: The tick-pathogen-vertebrate host interaction is probably much more complex than previously thought taking into account the frequently occurring events of co-infection, reinfection and superinfection. This complex situation could not be easily simulated in an experimental infection and underlines the value of field studies.

Keywords: Anaplasma phagocytophilum, Variant, Cattle, ankA, Multilocus sequence typing (MLST), Tick-borne fever, Germany, Pathogenicity, Roe deer

\section{Background}

Anaplasma phagocytophilum is a Gram-negative obligate intracellular bacterium that replicates in neutrophil granulocytes [1]. It is transmitted by ticks of the Ixodes ricinus

*Correspondence: friederike.loewenich@unimedizin-mainz.de ${ }^{1}$ Department of Medical Microbiology and Hygiene, University of Mainz, Obere Zahlbacherstrasse 67, 55131 Mainz, Germany

Full list of author information is available at the end of the article complex and causes febrile illness in humans and animals $[2,3]$. The disease entity in domestic ruminants such as sheep, cattle and goats, is referred to as tick-borne fever [4]. Main clinical signs in cattle consist in fever, inappetence, cough, lower limb oedema, drop in milk yield and abortion $[4,5]$. Leukopenia, thrombopenia and anemia are typical laboratory findings [6].

The incubation time to the development of clinical signs in needle-inoculated cattle varied between four

c) The Author(s) 2020. This article is licensed under a Creative Commons Attribution 4.0 International License, which permits use, sharing, adaptation, distribution and reproduction in any medium or format, as long as you give appropriate credit to the original author(s) and the source, provide a link to the Creative Commons licence, and indicate if changes were made. The images or other third party material in this article are included in the article's Creative Commons licence, unless indicated otherwise in a credit line to the material. If material is not included in the article's Creative Commons licence and your intended use is not permitted by statutory regulation or exceeds the permitted use, you will need to obtain permission directly from the copyright holder. To view a copy of this licence, visit http://creativecommons.org/licenses/by/4.0/. The Creative Commons Public Domain Dedication waiver (http://creativecommons.org/publicdomain/zero/1.0/) applies to the data made available in this article, unless otherwise stated in a credit line to the data. 
to eleven days [7-9] and A. phagocytophilum became detectable in the blood via nested PCR between days 3 and 6 post-infection (pi) [6]. The maximal bacteremic phase determined by nested PCR lasted between 17-26 days pi [6]. Bovine blood obtained after day 14 pi was not infectious any more for subinoculated cattle [7]. Without treatment, clinical recovery was observed in an experimental setting in an average of eight days [6] and under field conditions within one week [10].

Outbreaks of tick-borne fever in cattle mainly happened after introduction of tick-naïve or newly bought animals to tick-infested pastures [11-14]. Further, it has been shown that the infection of cattle with $A$. phagocytophilum occurred primarily at the beginning of the pasture season [15] and especially in heifers that grazed for the first time [10]. Although cattle seem to be affected only once per lactation period, immunity appears to be short lived because attacks in the successive lactation periods might occur [7, 11]. Even if the same strains were used as a homologous challenge in an experimental setting, some of the animals were susceptible to clinically apparent tick-borne fever in the six to twelve months following the primary infection $[7,8]$. However, in a recent comprehensive study of tick-borne fever in a German cattle herd over an entire pasture season of five months, five out of 15 initially infected heifers did not show clinical signs when they experienced re- or superinfection [10].

Previously, the $A$. phagocytophilum strains circulating in this herd were genetically characterized using the $16 S$ rRNA, groEL, $m s p 2$ and $m s p 4$ genes [10]. However, not all four loci were amplifiable in all animals due to technical problems. Therefore, each gene could only be used in terms of single locus analysis limiting the discriminatory power to distinguish distinct $A$. phagocytophilum strains. Thus, reinfection with the same strain or superinfection with a different genetic variant could not always be clearly distinguished. Further, it was unclear whether simultaneous co-infection with different genetic variants might have occurred because samples obtained at chronologically close collection dates were not considered for molecular characterization. For this reason, we chose multilocus sequence typing (MLST) [16] instead of single locus approaches and $a n k A$-based typing, which has been found in the past to have high discriminatory power [17]. All positive samples still available including those from chronologically close collection dates were analyzed.

The aim was (i) to investigate whether simultaneous co-infection with different $A$. phagocytophilum strains occurs under field conditions; (ii) to examine whether reinfection with the same strain takes place during one pasture season; (iii) to study whether superinfection with a second variant happens during the same pasture period; and (iv) to observe whether reinfection or superinfection lead to clinical signs.

Cattle on pasture are continuously exposed to ticks. Therefore, it is impossible in field studies to clearly distinguish between reinfection with the same strain or reactivation of latent infection. Similarly, it is impractical to definitely separate in-animal evolution of a variant from superinfection. Nevertheless, the results of the genetic characterization presented here and the findings from previous experimental infections helped to define criteria that are discriminatory at least to a certain extent. Here, we report the circulation of six A. phagocytophilum variants in varying combinations in a cattle herd over one pasture season. This complex situation could not be easily simulated in an experimental infection and underlines the value of field studies.

\section{Methods \\ Samples}

The blood samples were collected from a dairy cattle herd in North-Rhine-Westphalia, Germany as part of a study reported previously [10]. The stock consisted of 39 cows and 19 heifers (Bos taurus). The term 'heifer' is used synonymously for 'first calf heifer' throughout the article. The animals were cross-breeds of Red and Black Holstein Friesian and German Simmental in a closed breeding system. This implies that none of the animals was bought in addition from other farmers. The heifers went to the pasture for the first time, whereas the cows had grazed during the previous seasons. The samples investigated here were from the 15 heifers that tested positive for $A$. phagocytophilum DNA between May and October 2011 [10]. In total, 47 samples from different collections dates were included. Generally, blood samples of each animal were taken prior to pasturing in May 2011 and then every other month until January 2012. After an animal was PCR-positive, blood samples were taken weekly for the following 6-8 weeks and thereafter every other week for further 6-8 weeks. The rectal temperature was measured daily and a value of $\geq 39.5{ }^{\circ} \mathrm{C}$ was considered as fever. Blood samples were drawn weekly in febrile animals. Further, the heifers were observed by the farmer for reduced milk yield, discharge from eyes and nose, lower limb oedema and stiff walking.

\section{Sequencing}

DNA from EDTA-anticoagulated blood samples was extracted using the High Pure PCR Template Preparation Kit (Roche, Mannheim, Germany). Seven housekeeping genes (pheS, glyA, fumC, $m d h, \operatorname{sucA}$, dnaN and $\operatorname{atp} A$ ) were used for MLST as reported previously [16]. Clonal complexes (CC) were defined by sharing identical alleles at five of the seven loci with at least one other 
member of the group. The ankA gene was partially amplified and bidirectionally sequenced as described [16]. GenBank accession numbers are shown in Additional file 1: Table S1. The MLST profiles for samples without ambiguous nucleotides were submitted to the A. phagocytophilum isolates database hosted on PubMLST (https ://pubmlst.org/aphagocytophilum/).

\section{Definition of persistence, co-infection, reinfection and superinfection}

The maximal bacteremic phase in experimentally infected cattle lasted between 17-26 days pi as determined by nested PCR [6]. Therefore, the continuous presence of the same ST in consecutive samples within 30 days after the first positive PCR was interpreted as persistence of the initially infecting $A$. phagocytophilum strain. The co-infection with different $A$. phagocytophilum ank $A$ variants has been described in a bovine congenital infection [18]. Therefore, the presence of different $\mathrm{ST}$ in consecutive samples after the first positive PCR was defined as co-infection with distinct $A$. phagocytophilum strains. Reinfection with the same ST was considered when the same ST reoccurred later than 30 days after the primary infection and at least two negative PCR results were obtained in between. Superinfection with a new ST was supposed when it was detected after at least one negative PCR result.

\section{Phylogenetic analysis}

Sequences were codon-aligned by ClustalW applying the PAM (Dayhoff) matrix. Trees were constructed using the neighbor-joining (NJ) method with the Jukes-Cantor model and the complete deletion option in the program MEGA X version 10.0.5 [19]. Bootstrap analysis was conducted with 1000 replicates. Pairwise distances between the sequences were computed using the Jukes-Cantor matrix and applying the complete deletion option.

\section{Comparison of typing methods}

Adjusted Wallace coefficients [20] and Simpson's index of diversity [21] were calculated using the online tool available at: http://www.comparingpartitions.info/index .php?link=Tool.

\section{Results}

\section{Cow samples}

In total, 47 samples of different collection dates from 15 heifers were included. Animals were positive for A. phagocytophilum DNA between one to six times (Table 1). One heifer (cow 7) was not examined for clinical signs. All others, except one (cow 59), suffered at the time of the first positive PCR result from clinically apparent tick-borne fever. The animals were not specifically treated against the infection and recovered in average within one week [10]. Heifers remained asymptomatic after the first detection of $A$. phagocytophilum by PCR even if they experienced second infection.

MLST of the A. phagocytophilum strains infecting the cows In general, different sequences of a given locus (pheS, glyA, fumC, $m d h, \operatorname{sucA}, \operatorname{dnaN}, \operatorname{atp} A$ ) were ascribed a unique, but arbitrary allele number and each unique combination of alleles was assigned a sequence type (ST). Six different ST were found to infect the cattle herd (Table 1). In $9 \%(4 / 47)$ of the samples, ambiguous nucleotides were found in three to four loci. The ambiguous nucleotides could be resolved, because three of the animals (cow 22, cow 49 and cow 58) harbored the respective other ST before and after the time of the double infection, although formally the PCR products were sequenced directly without prior cloning. The nucleotide exchanges in the animals simultaneously harboring different ST are shown in Additional file 2: Table S2a and the ascribed allele numbers in Additional file 2: Table S2b.

ST 297 was a single locus variant (SLV) and ST 275 a double locus variant (DLV) of ST 243. Together, this three ST formed the new clonal complex (CC) 15 (Fig. 1). The novel CC 14 was constituted by ST 244 and by ST 21 which was formerly found in a European bison (Bison bonasus) [16]. ST 245 was part of CC 4 that has been described to contain samples from European bison and goat (Capra aegagrus hircus) [16]. Cow 59, which never developed clinical tick-borne fever, harbored ST 246 that belonged to the recently defined CC 13 . CC 13 was found to include samples from roe deer (Capreolus capreolus) and water buffalo (Bubalus bubalis) [22].

The concatenated nucleotide sequences (2877 bp) of ST 243, ST 244 and ST 245 differed by 4-13 single nucleotide polymorphisms (SNPs) and were highly identical to each other (Table 2). ST 297 infecting cow 57 on the 4th of September varied only by one SNP in the sucA locus from ST 243. However, the roe deer-associated ST 246 found in the asymptomatic cow 59 was more distantly related. It showed 76 to 78 SNPs when compared to the other five cattle-associated ST.

ank $A$ gene-based typing of the $A$. phagocytophilum strains infecting the cows

Four different $a n k A$ alleles were found to infect the cattle herd (Table 1). They were designated with letters (a, $\mathrm{b}, \mathrm{c}, \mathrm{d})$ to prevent confusion with the ank $A$ gene clusters described earlier [16]. ankA allele a had the highest prevalence with 53\% (25/47) (Fig. 2). In 9\% (4/47) ambiguous nucleotides were present in the ankA chromatograms. These were the same heifers that were simultaneously infected with two $A$. phagocytophilum ST at 
Table 1 Results of the genetic characterization of the A. phagocytophilum strains infecting the 15 heifers

\begin{tabular}{|c|c|c|c|c|c|c|}
\hline Cow no. & Date of positive PCR & ST & ankA allele & $\begin{array}{l}\text { Days between positive } \\
\text { PCR results }\end{array}$ & $\begin{array}{l}\text { Days between positive } \\
\text { PCR results }\end{array}$ & Interpretation \\
\hline \multirow[t]{2}{*}{7} & 19.06.2011 & 243 & a & na & na & na \\
\hline & 25.06.2011 & 243 & a & 6 & 6 & Persistence \\
\hline \multirow[t]{2}{*}{14} & 04.06 .2011 & 243 & a & na & na & na \\
\hline & 11.06 .2011 & 243 & a & 7 & 7 & Persistence \\
\hline \multirow[t]{4}{*}{22} & 30.05 .2011 & 243 & a & na & na & na \\
\hline & 04.06 .2011 & 243,244 & $a, b$ & 5 & 5 & Persistence, co-infection \\
\hline & 09.07.2011 & 244 & $b$ & 40 & 35 & Reinfection \\
\hline & 16.10.2011 & 245 & c & 139 & 99 & Superinfection \\
\hline 28 & 03.10 .2011 & 245 & c & na & na & na \\
\hline \multirow[t]{6}{*}{30} & 17.05.2011 & 244 & $b$ & na & na & na \\
\hline & 19.05.2011 & 244 & $b$ & 2 & 2 & Persistence \\
\hline & 25.05 .2011 & 243 & a & 8 & 6 & Co-infection \\
\hline & 30.05 .2011 & 243 & a & 13 & 5 & Persistence \\
\hline & 04.06 .2011 & 243 & a & 18 & 13 & Persistence \\
\hline & 25.06 .2011 & 275 & a & 39 & 21 & Superinfection \\
\hline \multirow[t]{3}{*}{35} & 17.05 .2011 & 243 & a & na & na & na \\
\hline & 19.05 .2011 & 243 & a & 2 & 2 & Persistence \\
\hline & 25.05 .2011 & 243 & a & 8 & 6 & Persistence \\
\hline \multirow[t]{2}{*}{36} & 19.05.2011 & 243 & a & na & na & na \\
\hline & 25.05 .2011 & 243 & a & 6 & 6 & Persistence \\
\hline \multirow[t]{6}{*}{46} & 22.05 .2011 & 243 & a & na & na & na \\
\hline & 25.05 .2011 & 243 & a & 3 & 3 & Persistence \\
\hline & 25.06 .2011 & 244 & $b$ & 34 & 31 & Superinfection \\
\hline & 03.07 .2011 & 244 & $b$ & 42 & 8 & Persistence \\
\hline & 17.07.2011 & 245 & c & 56 & 14 & Superinfection \\
\hline & 21.08 .2011 & 244 & $b$ & 91 & 35 & Reinfection \\
\hline \multirow[t]{4}{*}{49} & 19.05.2011 & 245 & c & na & na & na \\
\hline & 25.05.2011 & 245 & c & 6 & 6 & Persistence \\
\hline & 30.05 .2011 & 243,245 & $\mathrm{a}, \mathrm{c}$ & 11 & 5 & Persistence, co-infection \\
\hline & 11.06 .2011 & 243 & $\mathrm{a}$ & 23 & 12 & Persistence \\
\hline \multirow[t]{3}{*}{52} & 14.09.2011 & 245 & c & na & na & na \\
\hline & 18.09.2011 & 245 & c & 4 & 4 & Persistence \\
\hline & 25.09 .2011 & 245 & c & 11 & 7 & Persistence \\
\hline \multirow[t]{2}{*}{53} & 22.06 .2011 & 243 & a & na & na & na \\
\hline & 25.06 .2011 & 243 & a & 3 & 3 & Persistence \\
\hline \multirow[t]{3}{*}{57} & 04.06 .2011 & 243 & a & na & na & na \\
\hline & 11.06 .2011 & 243,244 & $a, b$ & 7 & 7 & Persistence, co-infection \\
\hline & 04.09 .2011 & 297 & a & 92 & 85 & Superinfection \\
\hline \multirow[t]{5}{*}{58} & 17.05 .2011 & 243 & a & na & na & na \\
\hline & 19.05.2011 & 243 & a & 2 & 2 & Persistence \\
\hline & 25.05.2011 & 243,245 & $\mathrm{a}, \mathrm{c}$ & 8 & 6 & Persistence, co-infection \\
\hline & 30.05 .2011 & 245 & c & 13 & 5 & Persistence \\
\hline & 09.07.2011 & 245 & c & 53 & 40 & Reinfection \\
\hline 59 & 04.09 .2011 & 246 & $d$ & na & na & na \\
\hline \multirow[t]{3}{*}{61} & 22.06 .2011 & 243 & a & na & na & na \\
\hline & 25.06 .2011 & 243 & a & 3 & 3 & Persistence \\
\hline & 09.07.2011 & 245 & c & 17 & 14 & Co-infection \\
\hline
\end{tabular}

${ }^{a}$ Referring to the first positive $\mathrm{PCR}$ result

${ }^{b}$ Referring to the previous positive PCR result Abbreviations: na, not applicable 
the respective time point (Additional file 2: Table S2). The ambiguous nucleotides could be resolved because three animals (cow 22, cow 49 and cow 58) harbored the respective other ankA allele before and after the time of the double infection, although formally the PCR products were sequenced directly without prior cloning.

The ank $A$ sequences (520 bp) of alleles a, b and c differed by 6-7 SNPs and were 99\% identical to each other (Table 3). However, ankA allele d found in the asymptomatic cow 59 was more distantly related. It showed 100 to 102 SNPs (without gaps) and had an identity of $80-81 \%$ when compared to the other three cattle-associated ank $A$ alleles.

\section{Concordance between typing methods}

Adjusted Wallace coefficients [20] were calculated to test for concordance between the different typing methods. The concordance between ST, CC and ankA allele was $100 \%$ when the 43 samples without ambiguous nucleotides were considered. The concordance between ankA and ST was still high ( $>75 \%$ ), but limited to $80 \%$ because ST 243, ST 275 and ST 297 shared ankA allele a (Table 4).

Earlier, the $A$. phagocytophilum strains circulating in the cattle herd were genetically characterized using the $16 S$ rRNA, groEL, msp 2 and $m s p 4$ genes [10]. However, not all loci were amplifiable in all samples and due to technical problems, some of the sequence reads were not of full length. Only two $16 S$ rRNA and two groEL gene variants were reported [10]. Therefore, the concordance between $16 S$ rRNA $(<10 \%)$ and groEL (55.5\%) gene-based typing and ST, CC and ankA allele was low (data not shown). Two $m s p 2$ variants were found previously in the cattle herd [10]. However, a full data set was only comparable in 11 samples yielding a concordance between $m s p 2$-based typing versus ST, CC and ankA allele of $100 \%$ (data not shown). Four msp4 types [10] from 19 samples could be used for comparison. The concordance between msp 4 versus $\mathrm{CC}$ and ankA allele was $100 \%$ (Table 4). The concordance between $m s p 4$ and ST was still high (>75\%), but limited to $77 \%$ because $m s p 4$ variant M4-49 [10] was shared by ST 243 and ST 297 (differing by one SNP in the sucA locus).

Simpson's index of diversity [21] was calculated as indication of the power of a typing method to distinguish between unrelated strains. It was determined in the 43 samples without ambiguous nucleotides. The Simpson's index of diversity was highest for ST, but this was not statistically significant because of overlapping $95 \%$ confidence intervals (Table 5).

\section{Persistence}

Our definition of persistence, co-infection, reinfection and superinfection is explained in the methods section. Six animals (cow 7, cow 14, cow 35, cow 36, cow 52 and cow 53) were infected with the same ST over time (Additional file 3: Table S3). The median presence of an identical ST in the blood was 7 days and the maximum presence 12 days.

\section{Co-infection}

The ankA gene probably undergoes recombination [23]. Therefore, it could bias the phylogenetic analysis and was not concatenated with the housekeeping genes used for MLST [16]. Here, the concordance between ST and ankA allele was $100 \%$ (Table 4). Therefore, the concatenation of the housekeeping genes with ankA did not disturb the interpretation and allowed the comparison of a longer sequence fragment.

Simultaneous co-infection with two ST occurred in six animals (cow 22, cow 30, cow 49, cow 57, cow 58 and cow 61). The co-infecting A. phagocytophilum strains differed by 14-18 SNPs when the 3397 bp (without gaps) of the concatenated housekeeping and ankA gene sequences were considered. The co-infecting strains were detected within a median interval of 6 days (Additional file 3: Table S3).

\section{Reinfection}

Three heifers (cow 22, cow 46 and cow 58) were reinfected with the same ST 35-40 days after the last positive PCR result (Additional file 3: Table S3). Heifers were positive by PCR only once when they acquired reinfection.

\section{Superinfection}

Superinfection with a new ST was observed in four heifers (cow 22, cow 30, cow 46 and cow 57) after a median free interval of 31 days after the last positive PCR result (Additional file 3: Table S3). Three negative PCR results were obtained in median between the detection of the

\footnotetext{
(See figure on next page.)

Fig. 1 Neighbour-joining (NJ) phylogenetic tree calculated from the concatenated housekeeping gene sequences. Tree construction was achieved by the NJ method using the Jukes-Cantor matrix with the complete deletion option. Bootstrap values are shown next to the branches. The scale-bar indicates the number of nucleotide substitutions per site. The final data set contained 2877 positions. Animals infected with only one ST are symbolized by black circles. Those harboring more than one ST over time are represented by green, red, yellow, purple, light blue, pink and dark blue circles. Ambiguous nucleotides in heifers with different ST at the same collection date were resolved before tree construction. Animals infected simultaneously with two different ST are represented by diamonds at the respective time point
} 


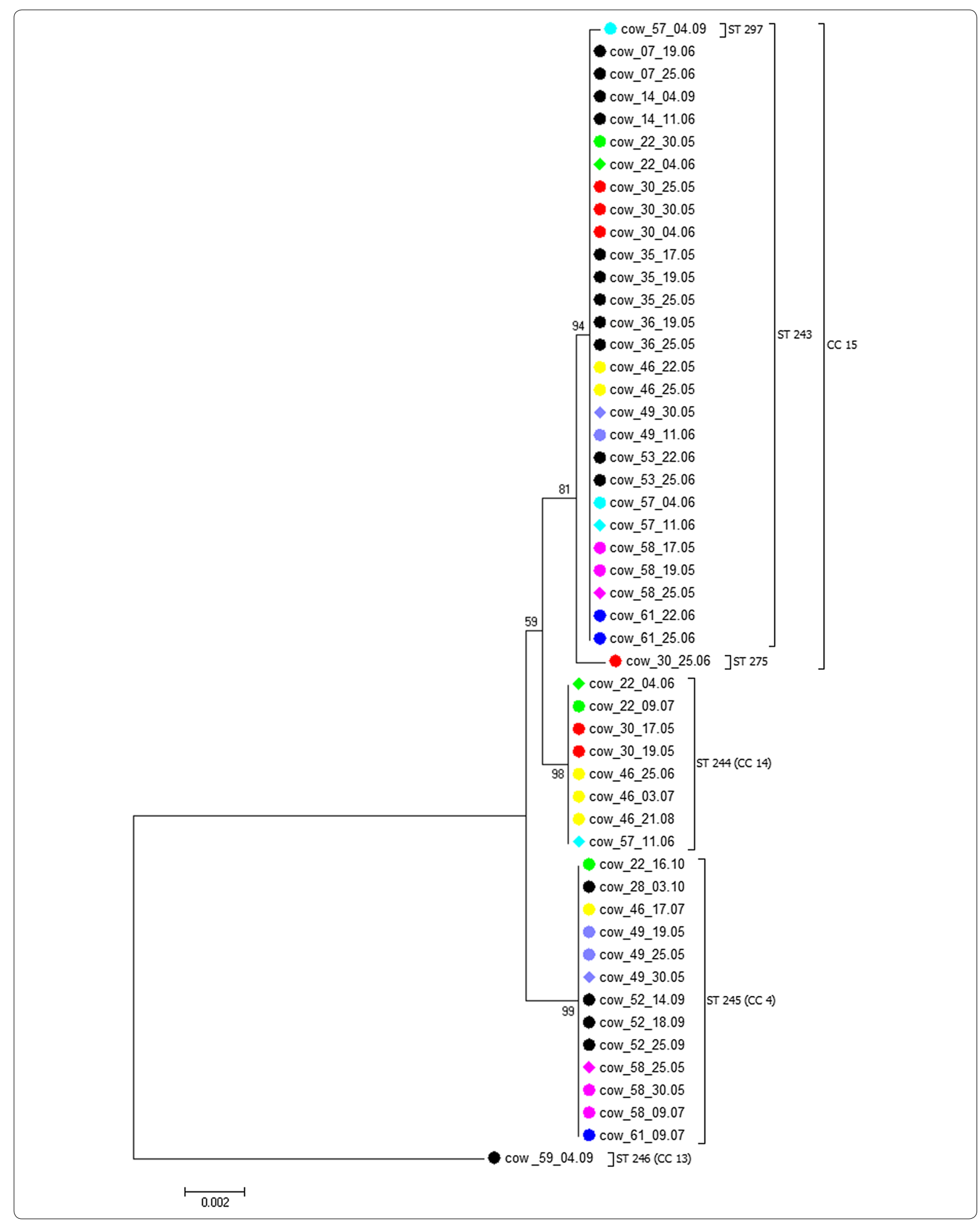


Table 2 SNPs (below the diagonal) and percent identity (above the diagonal) between the concatenated housekeeping gene sequences of the six ST infecting the cattle herd

\begin{tabular}{lllllll}
\hline & ST 243 & ST 244 & ST 245 & ST 246 & ST 275 & ST 297 \\
\hline ST 243 & & 99.76 & 99.62 & 97.31 & 99.86 & 99.69 \\
ST 244 & 7 & 8 & 99.72 & 97.35 & 99.55 & 99.72 \\
ST 245 & 11 & 76 & 76 & & 97.31 & 99.58 \\
ST 246 & 77 & 9 & 13 & 77 & 57.27 \\
ST 275 & 4 & 8 & 12 & 78 & 59.83 \\
ST 297 & 1 & & & & 5 \\
\hline
\end{tabular}

previous $A$. phagocytophilum strain and the superinfecting variant. Cow 46 was superinfected twice with different ST (Additional file 3: Table S3). The 3397 bp (without gaps) of the concatenated housekeeping and $a n k A$ gene sequences of the superinfecting strains differed by one (ST 297), four (ST 275) or 14 (ST 244, ST 245) SNPs from the variant detected before. Except for cow 46, which experienced superinfection with ST 244 lasting 8 days (Additional file 3: Table S3), heifers were positive by PCR only once when they acquired superinfection.

\section{Comparison to previously published housekeeping gene sequences}

The concatenated housekeeping gene sequences from our study were compared to 347 sequences without ambiguous nucleotides described previously [16, 22, 24-26]. The sequences were found in six clusters (Additional file 4: Fig. S1). Clusters 1, 2 and 3 were described earlier [16]. Cluster 1 contained sequences from humans, domestic animals (dogs, horses and cats), farm animals (cattle, sheep and goats), large wild animals (wild boars, European bison, red deer, chamois and red foxes), small mammals (hedgehogs) and I. ricinus ticks. Cluster 2 harbored samples from roe deer and I. ricinus ticks, but also sporadically sequences from domestic ruminants (three goats, one water buffalo and the asymptomatically infected cow 59). Cluster 3 was restricted to strains from rodents and shrews. The newly described Cluster 4 contained only three sequences (two roe deer and one red deer). Cluster 5 and Cluster 6 were originally described to be separate clades of Cluster 1 and 3, respectively [25] and harbored exclusively I. persulcatus and I. pavlovskyi ticks from the Asian part of Russia.

\section{Comparison to previously published ank $A$ gene sequences}

The ank $A$ sequences from our study were compared to 389 sequences without ambiguous nucleotides described previously $[16,22,26]$. The sequences were found in five clusters (Additional file 5: Fig. S2). described earlier [16]. Cluster 1 contained sequences from humans, domestic animals (dogs, horses and cats), farm animals (cattle, sheep and goats), large wild animals (wild boars, European bison, red deer, chamois and red foxes), small mammals (hedgehogs) and $I$. ricinus ticks. Cluster 2 harbored samples from roe deer and I. ricinus ticks, but also sporadically sequences from domestic ruminants (one goat, one water buffalo and the asymptomatically infected cow $59)$. Cluster 3 was, with the exception of two red deer strains, restricted to samples from roe deer. Cluster 4 contained strains from domestic ruminants (cattle, sheep and goats), wild ruminants (red deer, European bison, roe deer and chamois) and $I$. ricinus ticks. Cluster 5 was restricted to samples from rodents and shrews.

\section{Discussion}

MLST and $a n k A$-based typing were proven to be suitable to distinguish the $A$. phagocytophilum strains circulating in a German cattle herd over one pasture season because six ST and four ankA alleles were detected. Both methods had high discriminatory power (Table 5). In contrast to the former attempt [10], all eight loci were amplifiable and of full length. Previously, two $16 S$ rRNA, two groEL,

\section{(See figure on next page.)}

Fig. 2 Neighbour-joining (NJ) phylogenetic tree calculated from the ankA gene sequences. Tree construction was achieved by the NJ method using the Jukes-Cantor matrix with the complete deletion option. Bootstrap values are shown next to the branches. The scale-bar indicates the number of nucleotide substitutions per site. The final data set contained 520 positions. GenBank accession numbers are given after the sample designation. Animals infected with only one ankA allele are symbolized by black circles. Those harboring more than one ankA allele over time are represented by green, red, yellow, purple, light blue, pink and dark blue circles. Ambiguous nucleotides in heifers with different ankA allele at the same collection date were resolved before tree construction. Animals infected simultaneously with two different ankA alleles are represented by diamonds at the respective time point 


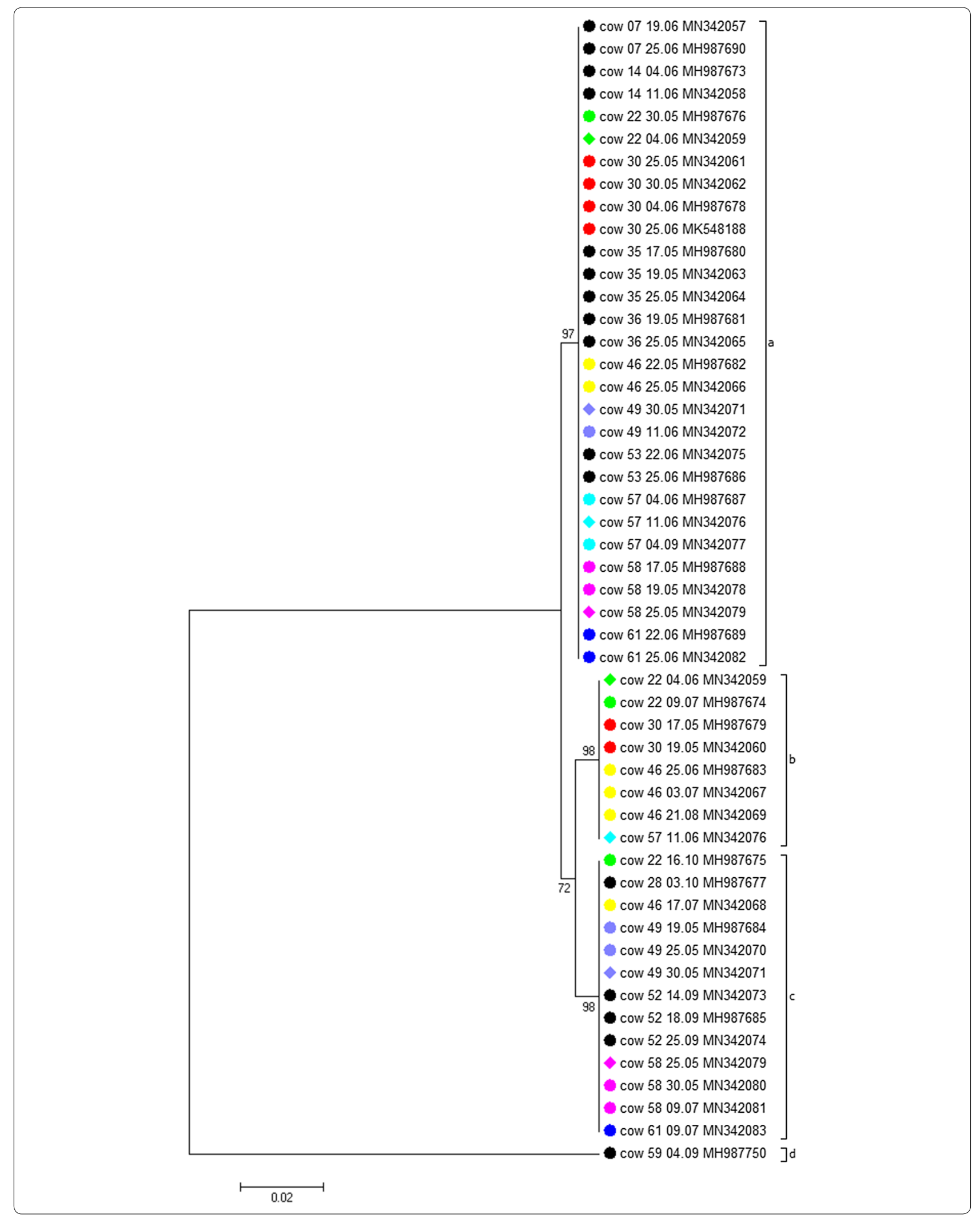


Table 3 SNPs (below the diagonal) and percent identity (above the diagonal) between ankA allele sequences of the four ankA alleles found in the cattle herd

\begin{tabular}{lllll}
\hline & $a$ & $b$ & $c$ & $d$ \\
\hline$a$ & & 98.64 & 98.64 & 80.82 \\
$b$ & 7 & & 98.84 & 80.32 \\
$c$ & 7 & 6 & & 80.32 \\
$d$ & 100 & 102 & 102 & \\
\hline
\end{tabular}

two $m s p 2$ and four $m s p 4$ variants were found in the herd [10]. Thus, only msp4-based typing was of comparable usefulness. However, single locus typing has an inferior resolution than multilocus typing [27]. The advantage of our MLST scheme is that it combines a standardized approach, a universe nomenclature and a free data exchange via the internet (https://pubmlst.org/aphag ocytophilum/).

The maximal bacteremic phase in experimentally infected cattle determined by nested PCR lasted between 17-26 days pi [6]. Considering an incubation time of 4-11 days [7-9], this is in line with our finding that the median presence of an identical A. phagocytophilum strain in the blood was seven days and the maximum presence 12 days.

Co-infection with two strains occurred in six animals. Their concatenated housekeeping and ankA gene sequences showed 14-18 SNPs. It seems unlikely that such an amount of SNPs arose via in-animal evolution because the new variant was detected within a median interval of six days after the first one. In sheep experimentally infected simultaneously with two different $16 \mathrm{~S}$ rRNA gene variants, both of them were detectable in their blood at day 0 [28]. Thereafter, depending on the proportion of both variants in the inoculum, only one of them was seen in peripheral blood. The second variant reappeared between four to 31 days pi. Thus, the initial detection of only one of both strains at the time of clinical presentation does not exclude co-infection. Formally, superinfection with a second variant within a medial interval of six days cannot be ruled out, although this
Table 5 Simpson's index of diversity calculated using the 43 samples from the cattle herd without ambiguous nucleotides

\begin{tabular}{lll}
\hline & Partitions & Simpson's index of diversity \\
\hline ST & 6 & $0.642(0.530-0.754)$ \\
CC & 4 & $0.590(0.473-0.707)$ \\
ankA & 4 & $0.590(0.473-0.707)$ \\
\hline \multicolumn{2}{l}{ Notes: The 95\% confidence intervals are given in parentheses }
\end{tabular}

seems less likely. The concept of co-infection with different $A$. phagocytophilum variants is supported by the fact that $41 \%(14 / 34)$ of $I$. ricinus ticks were infected with more than one ST [16]. Further, naturally infected cattle simultaneously harbored more than one A. phagocytophilum variant because double peaks were observed in the chromatograms of the genetic loci investigated [26, 29]. However, most animals were only sampled once, or the full profile of all loci was not available in these studies.

Reinfection with the same A. phagocytophilum strain occurred 35-40 days after the last positive PCR result. Cattle experimentally infected with A. phagocytophilum achieved pathogen clearance within 14 days as bovine blood obtained thereafter was not infectious any more for sub-inoculated cattle [7]. Thus, in our setting, reinfection seems to be more probable than reactivation of latent infection, although it is impossible in a field study to clearly distinguish between both of them. In contrast to cattle, needle-inoculated sheep housed under tick-free conditions experienced recurrent asymptomatic cycles of bacteremia after recovering from clinically overt tickborne fever [30]. The average negative period between two of these consecutive cycles was five days.

The concatenated housekeeping and ankA gene sequences of the superinfecting strains differed in most instances by 14 SNPs from the variant detected before. It is unlikely that this amount of SNPs arose by in-animal evolution. However, the single SNP that distinguishes ST 243 and the superinfecting ST 297 might have arisen by recent microevolution. The superinfection with a new $A$. phagocytophilum variant has been observed before in four cattle that became PCRpositive again after an interval of 7-17 months [29].

Table 4 Adjusted Wallace coefficients for ST, CC, ankA allele and msp4 variant

\begin{tabular}{lllll}
\hline & ST & CC & ankA & msp4 \\
\hline ST & & $1.000(1.000-1.000)$ & $1.000(1.000-1.000)$ & $1.000(1.000-1.000)$ \\
CC & $0.802(0.561-1.000)$ & & $1.000(1.000-1.000)$ & $1.000(1.000-1.000)$ \\
ankA & $0.802(0.561-1.000)$ & $1.000(1.000-1.000)$ & & $1.000(1.000-1.000)$ \\
msp4 & $0.772(0.411-1.000)$ & $1.000(1.000-1.000)$ & $1.000(1.000-1.000)$ & \\
\hline
\end{tabular}

Notes: Only samples without ambiguous nucleotides were considered. The $95 \%$ confidence intervals are given in parentheses 
However, it was not reported whether they experienced a second attack of clinically apparent tick-borne fever. Further, in contrast to our study, the animals were treated against tick-borne fever with oxytetracycline [29] probably biasing the results.

After the first attack, the heifers studied here were immune against clinically overt tick-borne fever for the entire pasture season of five months [10]. Accordingly, cattle were affected under field conditions only once per lactation period [7]. This is in contrast to the experimental setting where $11 / 14$ [7] or $2 / 5$ [8] animals were susceptible to clinically apparent tick-borne fever in the six to twelve months following the primary infection. The microscopic re-occurrence of $A$. phagocytophilum was reported in one of these two studies that both were done before PCR became available [8]. However, it is so far unknown whether the clinically immune animals experienced asymptomatic reinfection because only symptomatic cattle were investigated by blood smear. On the other hand, the microscopic detection of A. phagocytophilum is mostly associated with clinically apparent infection [10]. One explanation for the discrepancy between field and experimental conditions might be the fact that cattle on pasture are continuously re-exposed to the pathogen by what immunity is probably boosted.

Immunity was not sterile in the cattle herd studied here because animals were reinfected with the same or superinfected by a different strain. With one exception, the PCR was positive for only one further time in the seven other events of re- or superinfection. This could reflect semi-immunity that shortens the bacteremic phase and protects against clinically apparent tickborne fever.

Experimentally infected cattle were clinically immune against a homologous bovine $A$. phagocytophilum strain when challenged within three months after the primary infection [31]. This was not the case when a heterologous cattle strain was used. However, we observed under field conditions protection against clinically overt tick-borne fever when superinfection with a heterologous variant occurred. It is unclear so far which degree of genetic relatedness would be sufficient to confer cross-protection because the strains used previously were not characterized genetically [31].

One of the heifers did not develop apparent tick-borne fever at all. Its concatenated housekeeping and ankA sequences differed by 177-178 SNPs from all other strains. The respective variant was found together with roe deer samples in the phylogenetic analysis based on
MLST (Cluster 2) and ankA (Cluster 2) (Figs. 3, 4). It could be argued that roe-deer associated strains are less pathogenic for domestic ruminants because a similar observation was made previously in an asymptomatically infected goat [22]. However, an A. phagocytophilum strain from a water buffalo with typical signs of tick-borne fever was also found in the roe-deer-associated MLST and ankA gene Cluster 2 [22]. On the other hand, 98\% (62/63) available bovine housekeeping gene sequences from this and previous studies $[16,26]$ were part of MLST Cluster 1. Further, 82\% (58/71) of the ankA sequences from cattle were found in ankA Cluster 1 and $17 \%(12 / 71)$ in Cluster 4. Using a multilocus sequence approach, a similar observation was made [32]. Samples from cattle were found in three clusters. Two of them contained the majority of bovine sequences, whereas the third harbored all roe deer samples and only three genotypes from cattle. Thus, it was claimed that roe deer are probably not the reservoir host for bovine tick-borne fever [16, 32].

We describe here two new MLST clusters (5 and 6) that were originally published to be separate clades of Cluster 1 and Cluster 3, respectively [25]. They harbored exclusively I. persulcatus and I. pavlovskyi ticks from the Asian part of Russia. A similar observation was made recently as groEL haplotypes from $I$. persulcatus from Russia were found in the segregated groEL Cluster 4 [33].

\section{Conclusions}

To the best of our knowledge, we report for the first time a comprehensive molecular characterization of the $A$. phagocytophilum strains circulating in a cattle herd over one pasture season and provide evidence for the occurrence of co-infection, reinfection and superinfection. This was only possible, because, in contrast to previous studies, samples of close collection dates were included. Further, our analysis highlights that the tick-pathogenvertebrate host interaction is much more complex than previously thought. Whole blood from naturally infected animals was often used as inoculum in previous experimental infections. The respective A. phagocytophilum strains were not characterized at all [31] or only be means of $16 \mathrm{~S}$ rRNA gene-based typing [28]. The low discriminatory power of the $16 \mathrm{~S}$ rRNA gene and the fact that field samples may contain not only a single $A$. phagocytophilum variant, limits the conclusions drawn from such studies. Therefore, a reliable and easily comparable typing method such as MLST should be preferably used for genetic characterization. 


\section{Supplementary information}

Supplementary information accompanies this paper at https://doi. org/10.1186/s13071-020-04032-2.

Additional file 1: Table S1. GenBank accession numbers of the housekeeping and ankA gene sequences from the 47 samples from the 15 heifers.

Additional file 2: Table S2. a Nucleotide exchanges in five of the seven housekeeping genes (phes, glyA, fumC, sucA, dnaN) and the ankA gene found in the four animals with simultaneous double infection with two different A. phagocytophilum strains. b Allel designation and ST found in the four animals with simultaneous double infection with two different $A$. phagocytophilum strains.

Additional file 3: Table S3. Detection of A. phagocytophilum by PCR for all collection dates in the 15 heifers and results of the genetic characterization.

Additional file 4: Figure S1. Neighbour-joining (NJ) phylogenetic tree calculated from the concatenated housekeeping gene sequences of the 43 cattle samples without ambiguous nucleotides and 347 samples without ambiguous nucleotides described previously. Tree construction was achieved by the NJ method using the Jukes-Cantor matrix with the complete deletion option. Bootstrap values $\geq 86 \%$ are shown. The scalebar indicates the number of nucleotide substitutions per site. The final data set contained 2877 positions. Identical ST are displayed only once per species. The number in parenthesis indicates the frequency with which the respective ST was found. Key: red circles, sequences from humans, dogs, horses and cats; dark blue diamonds, sequences from domestic ruminants; light blue diamonds, sequences from wild ruminants; green triangles, sequences from small mammals; yellow squares, sequences from wild boars; purple triangles, sequences from red foxes; white triangles, sequences from ticks.

Additional file 5: Figure S2. Neighbour-joining (NJ) phylogenetic tree calculated from the ankA gene sequences of the 43 cattle samples without ambiguous nucleotides and 389 samples without ambiguous nucleotides described previously. Tree construction was achieved by the $\mathrm{NJ}$ method using the Jukes-Cantor matrix with the complete deletion option. Bootstrap values $\geq 81 \%$ are shown. The scale-bar indicates the number of nucleotide substitutions per site. The final data set contained 516 positions. Identical ankA sequences are displayed only once per species. GenBank accession numbers are given after the species designation. The number in parenthesis indicates the frequency with which the respective sequences was found. Key: red circles, sequences from humans, dogs, horses and cats; dark blue diamonds, sequences from domestic ruminants; light blue diamonds, sequences from wild ruminants; green triangles, sequences from small mammals; yellow squares, sequences from wild boars; purple triangles, sequences from red foxes; white triangles, sequences from ticks.

\section{Abbreviations}

CC: clonal complex; MLST: multilocus sequence typing; J: neighbor-joining; SNP: single nucleotide polymorphism; ST: sequence type.

\section{Acknowledgements}

The work presented here is part of the MD thesis of Denis B. Langenwalder.

\section{Authors' contributions}

CS, MN and MP designed the study. DL and FVL did the genetic characterization. FvL performed the phylogenetic analyses and wrote the manuscript. All authors read and approved the final manuscript.

\section{Funding}

Not applicable.

\section{Availability of data and materials}

All data generated or analysed during this study are included in this published article and its additional files. All newly generated nucleotide sequences were submitted to the GenBank database under the accession numbers shown in
Additional file 1: Table S1. The MLST profiles for samples without ambiguous nucleotides were submitted to the A. phagocytophilum isolates data base hosted on PubMLST (https://pubmlst.org/aphagocytophilum/).

\section{Ethics approval and consent to participate}

The blood samples were taken as part of the diagnostic workup of a previous study [10]. Thus, ethical approval was not necessary. Written informed consent was obtained from the owner of the cattle.

\section{Consent for publication}

Not applicable.

\section{Competing interests}

The authors declare that they have no competing interests.

\section{Author details}

${ }^{1}$ Department of Medical Microbiology and Hygiene, University of Mainz, Obere Zahlbacherstrasse 67, 55131 Mainz, Germany. ${ }^{2}$ Institute of Infectology, Friedrich-Loeffler-Institut, Südufer 10, 17493 Greifswald-Insel Riems, Germany. ${ }^{3}$ Institute for Animal Hygiene and Veterinary Public Health, University of Leipzig, An den Tierkliniken 1, 04103 Leipzig, Germany.

Received: 18 October 2019 Accepted: 24 March 2020

Published online: 30 March 2020

\section{References}

1. Dumler JS, Barbet AF, Bekker CPJ, Dasch GA, Palmer GH, Ray SC, et al. Reorganization of genera in the families Rickettsiaceae and Anaplasmataceae in the order Rickettsiales: unification of some species of Ehrlichia with Anaplasma, Cowdria with Ehrlichia and Ehrlichia with Neorickettsia, descriptions of six new species combinations and designation of Ehrlichia equi and 'HGE agent' as subjective synonyms of Ehrlichia phagocytophila. Int J Syst Evol Microbiol. 2001;51:2145-65.

2. Stuen S, Granquist EG, Silaghi C. Anaplasma phagocytophilum-a widespread multi-host pathogen with highly adaptive strategies. Front Cell Infect Microbiol. 2013;3:31.

3. Ismail N, McBride JW. Tick-borne emerging infections: ehrlichiosis and anaplasmosis. Clin Lab Med. 2017;37:317-40.

4. Woldehiwet Z. The natural history of Anaplasma phagocytophilum. Vet Parasitol. 2010;167:108-22.

5. Brun-Hansen $\mathrm{H}$, Grønstøl H, Hardeng F. Experimental infection with Ehrlichia phagocytophila in cattle. Zentralbl Veterinarmed B. 1998;45:193-203.

6. Pusterla N, Huder J, Wolfensberger C, Braun U, Lutz H. Laboratory findings in cows after experimental infection with Ehrlichia phagocytophila. Clin Diagn Lab Immunol. 1997:4:643-7.

7. Hudson JR. The recognition of tick-borne fever as a disease of cattle. Brit Vet J. 1950;106:3-17.

8. Tuomi J. Experimental studies on bovine tick-borne fever. 1. Clinical and haematological data, some properties of the causative agent, and homologous immunity. Acta Pathol Microbiol Scand. 1967;70:429-45.

9. Pusterla N, Braun U. Clinical findings in cows after experimental infection with Ehrlichia phagocytophila. Zentralbl Veterinarmed A. 1997:44:385-90.

10. Silaghi C, Nieder M, Sauter-Louis C, Knubben-Schweizer G, Pfister K, Pfeffer M. Epidemiology, genetic variants and clinical course of natural infections with Anaplasma phagocytophilum in a dairy cattle herd. Parasit Vectors. 2018;11:20.

11. Foggie A, Allison CJ. A note on the occurence of tick-borne fever in cattle in Scotland with comparative studies of bovine and ovine strains of the organism. Vet Rec. 1960;72:767-70.

12. Wilson JC, Foggie A, Carmichael MA. Tick-borne fever as a cause of abortion and stillbirths in cattle. Vet Rec. 1964;76:1081-4.

13. Cranwell MP, Gibbons JA. Tick-borne fever in dairy herd. Vet Rec. 1986:119:531-2.

14. Guyot H, Ramery E, O'Grady L, Sandersen C, Rollin F. Emergence of bovine ehrlichiosis in Belgian cattle herds. Ticks Tick Borne Dis. 2011:2:116-8.

15. Pusterla N, Pusterla JB, Braun U, Lutz H. Serological, hematologic, and PCR studies of cattle in an area of Switzerland in which tick-borne fever 
(caused by Ehrlichia phagocytophila) is endemic. Clin Diagn Lab Immunol. 1998;5:325-7.

16. Huhn C, Winter C, Wolfsperger T, Wüppenhorst N, Strašek Smrdel K, Skuballa J, et al. Analysis of the population structure of Anaplasma phagocytophilum using multilocus sequence typing. PLoS One. 2014;9:e93725.

17. Scharf W, Schauer S, Freyburger F, Petrovec M, Schaarschmidt-Kiener D, Liebisch G, et al. Distinct host species correlate with Anaplasma phagocytophilum ankA gene clusters. J Clin Microbiol. 2011;49:790-6.

18. Henniger T, Henniger P, Grossmann T, Distl O, Ganter M, von Loewenich FD. Congenital infection with Anaplasma phagocytophilum in a calf in northern Germany. Acta Vet Scand. 2013;55:38.

19. Kumar S, Stecher G, Li M, Knyaz C, Tamura K. MEGA X: Molecular Evolutionary Genetics Analysis across computing platforms. Mol Biol Evol. 2018:35:1547-9.

20. Severiano A, Pinto FR, Ramirez M, Carriço JA. Adjusted Wallace coefficient as a measure of congruence between typing methods. J Clin Microbiol. 2011;49:3997-4000

21. Hunter PR, Gaston MA. Numerical index of the discriminatory ability of typing systems: an application of Simpson's index of diversity. J Clin Microbiol. 1988;26:2465-6.

22. Langenwalder DB, Schmidt S, Gilli U, Pantchev N, Ganter M, Silaghi C, et al. Genetic characterization of Anaplasma phagocytophilum strains from goats (Capra aegagrus hircus) and water buffalo (Bubalus bubalis) by 165 rRNA gene, ankA gene and multilocus sequence typing. Ticks Tick Borne Dis. 2019;10:101267.

23. Majazki J, Wüppenhorst N, Hartelt K, Birtles R, von Loewenich FD. Anaplasma phagocytophilum strains from small mammals exhibit specific ankA gene sequences. BMC Vet Res. 2013;9:235.

24. Tveten AK. Prevalence and diversity among Anaplasma phagocytophilum strains originating from /xodes ricinus ticks from northwest Norway. J Pathog. 2014;2014:824897.

25. Mukhacheva TA, Shaikhova DR, Kovalev SY. Asian isolates of Anaplasma phagocytophilum: multilocus sequence typing. Ticks Tick Borne Dis. 2019;10:775-80.
26. Tegtmeyer P, Ganter M, von Loewenich FD. Simultaneous infection of cattle with different Anaplasma phagocytophilum variants. Ticks Tick Borne Dis. 2019;10:1051-6.

27. Dugat T, Lagreé AC, Maillard R, Boulouis HJ, Haddad N. Opening the black box of Anaplasma phagocytophilum diversity: current situation and future perspectives. Front Cell Infect Microbiol. 2015;5:61.

28. Stuen S, Dahl H, Bergström K, Moum T. Unidirectional suppression of Anaplasma phagocytophilum genotypes in infected lambs. Clin Diagn Lab Immunol. 2005;12:1448-50.

29. Lagrée AC, Rouxel C, Kevin M, Dugat T, Girault G, Durand B, et al. Cocirculation of different $A$. phagocytophilum variants within cattle herds and possible reservoir role for cattle. Parasit Vectors. 2018;11:163.

30. Thomas RJ, Birtles RJ, Radford AD, Woldehiwet Z. Recurrent bacteraemia in sheep infected persistently with Anaplasma phagocytophilum. J Comp Pathol. 2012;147:360-7.

31. Tuomi J. Experimental studies on bovine tick-borne fever. 3. Immunological strain differences. Acta Pathol Microbiol Scand. 1967;71:89-100.

32. Chastagner A, Dugat T, Vourc'h G, Verheyden H, Legrand L, Bachy V, et al. Multilocus sequence analysis of Anaplasma phagocytophilum reveals three distinct lineages with different host ranges in clinically ill French cattle. Vet Res. 2014;45:114.

33. Jaarsma Rl, Sprong H, Takumi K, Kazimirova M, Silaghi C, Mysterud A, et al. Anaplasma phagocytophilum evolves in geographical and biotic niches of vertebrates and ticks. Parasit Vectors. 2019;12:328.

\section{Publisher's Note}

Springer Nature remains neutral with regard to jurisdictional claims in published maps and institutional affiliations.
Ready to submit your research? Choose BMC and benefit from:

- fast, convenient online submission

- thorough peer review by experienced researchers in your field

- rapid publication on acceptance

- support for research data, including large and complex data types

- gold Open Access which fosters wider collaboration and increased citations

- maximum visibility for your research: over $100 \mathrm{M}$ website views per year

At $\mathrm{BMC}$, research is always in progress.

Learn more biomedcentral.com/submissions 